\title{
Marcadores genéticos (H LA) y perfil de auto-anticuerpos en una familia mapuche con un caso de diabetes tipo 1
}

\author{
Sylvia Asenjo $\mathrm{M}^{\mathbf{1}}$, Andrea G leisner $\mathrm{E}^{1}$, Francisco Pérez $\mathrm{B}^{2 \mathrm{a}}$. \\ HLA genetic markers and auto-antibody \\ profile in a Mapuche family with a case \\ affected of type 1 diabetes
}

Background: Type 1 diabetes (DM1) is caused by an autoimmune process that destroys beta cells of pancreas. Not all carriers of susceptible HLA genes and positive for autoantibodies develop the disease. Environmental factors play a role in triggering the autoimmune process. Aim: To analyze an exceptional case of DM1 in a Mapuche family in the context of genetic, immunological and environmental factors. Subjects and methods: A study of a family with an affected female child was carried out in a Mapuche community in Southern Chile (VIII region). This is an unique and sporadic DM1 case with Mapuche heritage. Nutritional and viral infections data were collected by interview and clinical records. A genetic analysis by PCR was done to detect class I and II HLA genes by reverse dot blot. Results: The proband, her mother and sister had positive islet cell antibodies (ICA). Her father and brother were negative. All the family was positive for anti glutamic decarboxylase antibodies (GAD65). All subjects had HLA-DRB1 0407/0407 and HLA-DQB1 0302/0302 alleles. The index case and her father were homozygotes for the HLA-A1:A*68012/A*68012 allele. Mean breast feeding lapse was 18 months in all children. No evidences for viral infections such as rubella, mumps or measles were found in this family. Conclusions: There was an altered profile of autoantibodies in the family of the index case. All genotypes were comparable with the European population where the diabetogenic combination DR4/DQB1*0302 is the most prevalent. No environmental factors could be incriminated as triggers of the disease (Rev Méd Chile 2004; 132: 47-50).

(Key Words: Diabetes Mellitus, insulin dependent, Ethnic groups; HLA-DR antigens).

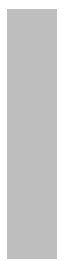

Recibido el 29 de mayo, 2003. Aceptado en versión corregida el 4 de septiembre, 2003.

Estudio financiado parcialmente por los Proyectos FONDECYT \#1000944 y MECESUP, Universidad de Chile PR005.

${ }^{1}$ Unidad de Endocrinología Pediátrica, Facultad de Medicina, Universidad de Concepción. ${ }^{2}$ Programa de Epidemiología Genética, Instituto de Nutrición y Tecnología de los Alimentos (INTA), Universidad de Chile.

a Doctor en Ciencias.

L a diabetes tipo 1 (DM1) constituye una de las enfermedades crónicas más comunes de la población infantil ${ }^{1,2}$. El registro epidemiológico de

Correspondencia a: Dra. Sylvia Asenjo M. Unidad de Endocrinología Pediátrica, Facultad de Medicina, Universidad de Concepción, Concepción, Chile. E mail: sasenjo@udec.cl
DM1 en Santiago, reveló una incidencia del orden de 2,49/100.000 hab/año para el período 1986$1993^{3}$, con posterioridad, durante los años 19971998 se ha observado una cifra de 4,1/100.000 hab/año ${ }^{4}$. Estos datos confirman, que al igual que en el resto del mundo, la diabetes tipo 1 también ha aumentado en nuestro país, de modo que Chile 
ha comenzado a ser considerado como un país de incidencia intermedia en esta patología. En la región metropolitana ésta fue de 4,58-3,9/100.000 hab/año en los años 1999-2000, respectivamente.

La diabetes tipo 1 autoinmune (Tipo $1 \mathrm{~A}$ de acuerdo a la nueva clasificación de la Asociación Americana de Diabetes, ADA 1997) es la resultante de un complejo proceso inmunológico donde es posible describir diversos niveles de respuesta, tanto de tipo celular como humoral, de severidad variable, que conduce a la destrucción de la célula ß pancreática en individuos genéticamente susceptibles 5 .

Durante el proceso de prediabetes, existe una serie de auto-anticuerpos dirigidos contra distintas estructuras de la célula beta que prestan utilidad como marcadores de un proceso necrótico que comienza a sufrir el tejido $\lesssim$ celular. Entre ellos podemos reconocer los anticuerpos contra células del islote (ICA), anticuerpos anti-insulina (IAA), anticuerpos anti-ácido glutámico decarboxilasa (GAD65) y anticuerpos anti-tirosina fosfatasa (IA$2)$, todos ellos útiles al momento de describir los cambios que se producen a nivel de respuesta inmunológica en la diabetes tipo $1^{6}$.

Por otra parte, desde el punto de vista genético, no todos los portadores de genes de susceptibilidad, e incluso no todos los individuos que presenten positividad contra alguno de estos anticuerpos pueden desarrollar diabetes tipo 1, lo que sugiere inequívocamente que existen factores ambientales que juegan un rol fundamental en el proceso destructivo de la célula beta. Existe amplia evidencia del efecto protector que tendría un período prolongado de lactancia matema exclusiva, la cual disminuina el impacto de antígenos ambientales introducidos mediante la lactancia y alimentación artificial ${ }^{7}$.

La composición étnica chilena está constituida por una mezcla variable de genes europeos y genes amerindios, lo que la transforma así, en una población interesante desde el punto de vista epidemiológico, que permitiría evaluar el posible impacto de la diversidad genética en la mayor 0 menor frecuencia de diabetes entre la población ${ }^{8}$. La etnia mapuche, representa el grupo autóctono mayoritario de nuestro país y corresponde al que tiene la mayor influencia en la constitución de la población chilena. Las investigaciones relacionadas a la diabetes tipo 1 en este grupo étnico, indican que presentan una bajísima incidencia de diabetes tipo 1, con cifras que se encuentran entre las más bajas del mundo 0,4/100.000 hab/año ${ }^{9,10}$.

Esta comunicación da a conocer la ocurrencia de un debut de diabetes tipo 1 de origen mapuche en la VIII región, considerado el único caso diagnosticado en esta etnia en los últimos 20 años, proveniente de una zona rural y donde se ha analizado al grupo familiar desde el punto de vista genético, inmunológico y ambiental. La VIII región tiene una población mapuche aproximada a 122.323 individuos mayores de 14 años según estimación del último censo.

\section{DiseÑo Y METODOLOGÍA}

Estudio familiar: Un total de 5 individuos fueron analizados durante el año 1999. El caso de diabetes tipo 1 correspondió a una niña de 10 años de edad, que debutó al año de vida, su hermana de 7 y un hermano de 11 años, respectivamente. El estudio incluyó a ambos padres, la madre de 35 y el padre de 38 años, todos ellos residentes de una comunidad indígena, de la VIII región. Las características de etnia mapuche estuvieron dadas por el análisis de apellidos en la familia (se analizaron hasta cuatro generaciones anteriores), caracteres antropométricos y análisis de grupo sanguíneo (ABO). Es el único caso clínico de diabetes tipo 1 conocido de etnia mapuche en los últimos 20 años. Ambos padres entregaron su consentimiento escrito de participación, siendo este un screening aprobado por el Comité de Etica Local.

Registro de patología viral y dieta de la infancia: La información concerniente a la nutrición infantil y antecedentes mórbidos, se obtuvo directamente de la madre mediante entrevista personal y de datos aportados por la historia clínica. Se registró la duración promedio de lactancia materna y antecedentes sobre patología viral asociada a la diabetes tipo 1, como rubéola, parotiditis y varicela en todo el grupo familiar.

Análisis genético: El DNA genómico de todos los individuos se extrajo mediante técnicas estándares a partir de linfocitos periféricos; $0,5 \mu \mathrm{g}$ de DNA fueron sometidos a una reacción de PCR para amplificar en forma específica regiones del complejo mayor de histocompatibilidad e identificar 
alelos de la región HLA de clase I (A, B, C, D y E) y de clase II: HLA-A, HLA-DRB1, HLA-DRB4 y HLA-DQB1 mediante dot blot reverso ${ }^{11}$.

Perfil de auto-anticuerpos: La determinación de los niveles séricos de anticuerpos se llevó a cabo empleando inmunofluorescencia indirecta sobre páncreas humano, clasificándose como (+) un título igual a $20 \mathrm{JDF}$ y $(+)$ a un título mayor a las $40 \mathrm{JDF}$. Los niveles de GAD65 se cuantificaron empleando un ELISA semicuantitativo (Biomerica, USA).

\section{RESULTADOS}

Los resultados obtenidos en este análisis mostraron como característica relevante una alta proporción de positividad de auto-anticuerpos en toda la familia. A lo menos un auto-anticuerpo fue positivo en cada integrante del grupo familiar: GAD65 fue positivo en todos ellos, e ICA se encontró positivo en la madre, el caso índice y su hermana. Todos los hijos recibieron lactancia materna exclusiva prolongada, en promedio 18 meses. No existen antecedentes de patología viral asociada a DM1, el registro fue negativo para rubéola, parotiditis y varicela. Desde el punto de vista genético, se observó una distribución homocigota idéntica en los padres e hijos para los alelos HLA-DRB1 (todos HLA-DRB1*0407 y HLA-DQB1*0302). Para la región HLA-A1 el caso afectado de diabetes tipo 1 resultó homocigoto para el alelo 68012, genotipo similar sólo al padre, dado que los demás miembros de la familia resultaron heterocigotos para este alelo. Sólo la combinación alélica del HLA de clase I B/D estuvo presente en el caso índice (Figura 1).

\section{Discusión}

La población mapuche de Chile presenta una bajísima incidencia de diabetes tipo 1, datos que han sido dados a conocer en estudios previos y que se confirman al existir un registro mínimo de nuevos casos de etnia mapuche afectada de esta enferme$\operatorname{dad}^{10}$. La familia analizada en esta investigación ha mostrado una genética de HLA de clase II idéntica entre todos sus componentes y similar a la mayor frecuencia que describen diversos estudios en relación a los alelos HLA-DRB1*0407 y HLADQB1*0302 ${ }^{12,13}$. Estos dos alelos han sido ampliamente asociados a diabetes tipo 1 en población de ongen caucasoide, no obstante ser alelos bastante frecuentes en grupos indígenas ${ }^{14}$. Respecto al alelo HLA-A1*68012 éste ha sido descrito en poblaciones abonígenes Guaraní, Toba y Wichi y es considerado un alelo fundador en grupos amerindios de Latinoamérica $^{15}$. Es muy llamativa la alta frecuencia observada de auto-anticuerpos positivos en la totalidad de la familia, indicando la presencia de un fuerte componente de reactividad beta pancreática, del cual no se tiene especial claridad, ya que todos sus integrantes poseen a lo menos un anticuerpo positivo ${ }^{16}$. No existen evidencias contundentes que permi-

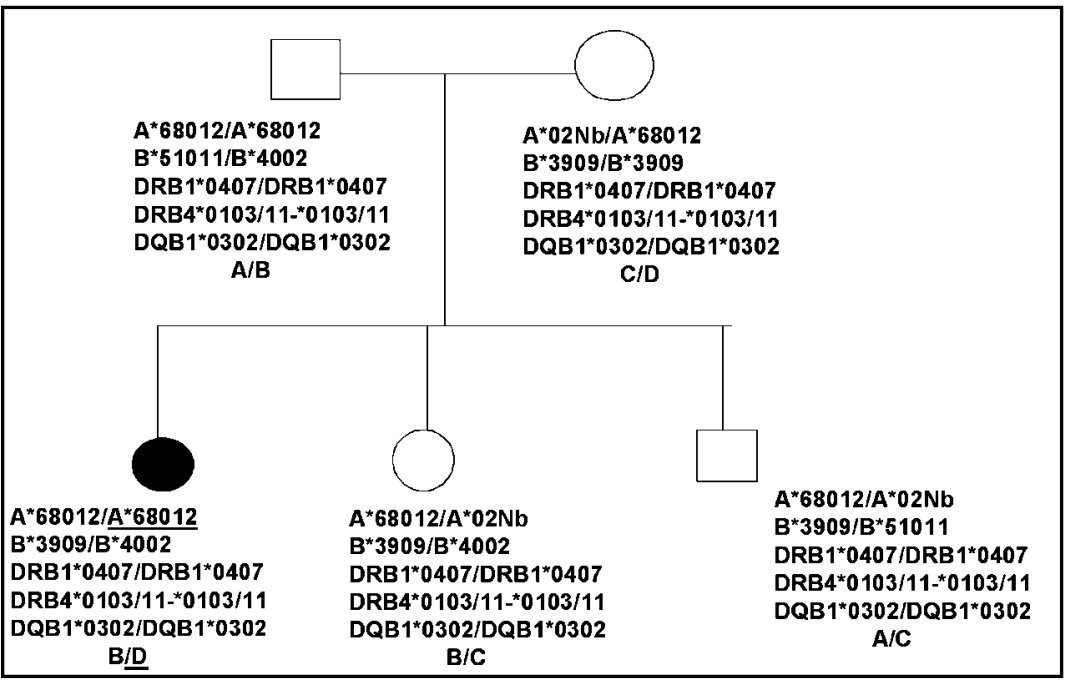

FIGURA 1. Genotipos HLA clase II y clase I en la familia mapuche. 
tan asociar este extraño caso de diabetes tipo 1 en esta etnia con algún componente ambiental, ya que los datos obtenidos en terreno indican claramente que se tratańa de un grupo protegido contra esta patología, ya sea por una nula frecuencia de patología viral asociada a DM1 como por un largo período de lactancia materna exclusiva. El futuro análisis de otros componentes del sistema HLA extendido o factores

\section{REFERENCIAS}

1. Dorman JS, BunKer CH. HLA-DQ locus of the human leukocyte antigen complex and type 1 diabetes mellitus: a HuGE review. Epidemiol Rev 2000; 22: 218-27.

2. AкERBLom HK, KNip M, Hyoty $H$, Rejonen $H$, VirTanen S, SaVilahti E et al. Interaction of genetic and environmental factors in the pathogenesis of insulin-dependent diabetes mellitus. Clin Chim Acta 1997; 257: 143.

3. Carrasco E, Pérez-Bravo F, Santos JL, López G, Calvilía M, Wolff C et al. One of the lowest validated incidence rates of insulin dependent diabetes mellitus in the Americas: Santiago, Chile. Diab Res Clin Pract 1996; 34 (Suppl): 153-7.

4. Santos JL, Carrasco E, Moore AL, Pérez-Bravo F, Albala C. Incidence rate and spatio-temporal clustering of type 1 diabetes in Santiago, Chile, from 1997 to 1998. Rev Saude Publica 2001; 35: 96-100.

5. Sonderstrup G, McDevitT HO. DR, DQ and you: MHC alleles and autoimmunity. J Clin Invest 2001; 107: 795-6

6. LesLe RDG, AtKinson MA, Notkins AL Autoantigens IA-2 and GAD in type 1 (insulin-dependent) diabetes. Diabetologia 1999; 42: 3-21.

7. Akerblom HK, Vaarala O, Hyoty H, Ilonen J, Knip M. Environmental factors in the etiology of type 1 diabetes. Am J Med Genet 2002; 115: 18-29.

8. ValenZuela C, AcuÑa M, Harb Z. Gradiente sociogenético de la población chilena. Rev Méd Chile 1987; 115: 295-9.

9. Larenas G, Arias G, Espinoza O, Charles M, Landaeta O, Vilinanueva S ET al. Prevalence of diabetes mellitus in an indigenous (Mapuche) community of the IX region in Chile. Rev Méd Chile 1985; 113: 1121-5.

10. Larenas G, Montecinos A, Manosalya A, Barthou M, VIDAL T. Incidence of insulin-dependent diabetes mellitus in the IX Region of Chile: Ethnic Differences. Diab Res Clin Pract 1996; 34 (Suppl): 147-51. genéticos no HLA ${ }^{17}$, podrían aportar mayor información respecto al proceso inmunológico que se manifiesta en esta familia, con un alto grado de auto-anticuerpos circulantes y una presencia de marcadores genéticos comunes en una población autóctona cuya incidencia de diabetes tipo 1 es casi nula y que evidentemente se encuentra protegida del desamollo de esta enfermedad.

11. Pérez-Bravo F, Carrasco E, Gutiérrez-López MD, Martínez MT, López G, García de los Ríos M. Genetic predisposition and environmental factors leading to the development of insulin-dependent diabetes mellitus in Chilean children. J Mol Med 1996; 74: 105-9.

12. Blagitiko N, O'hUigin C, Figueroa F, Horai S, Sonoda S, Tajima K et al. Polymorphism of the HLA-DRB1 locus in Colombian, Ecuadorian and Chilean Amerinds. Hum Immunol 1997; 54: 7481.

13. Sonoda S, Arce-Gómez B, Satz ML, Gorodezky C, Juárez V, Olvo A ET AL. Ethnic report on native americans in South America and Mexico. In: Tsuji K, Aizawa M Eds. HLA 1991: Proceedings of the XI International Histocompatibility Workshop and Conference. Oxford Science Publications 1992; 685-8.

14. Serrano-Ríos M, Goday A, Martínez-Larrad MT. Migrant populations and the incidence of type 1 diabetes mellitus: an overview of the literature with a focus on the Spanish-Heritage countries in Latin America. Diab Metab Res Rev 1999; 15: 113-32.

15. CADAVID LF, WatKINS DI. Heirs of the Jaguar and the Anaconda: HLA, conquest and disease in the indigenous populations of the Americas. Tissue Antigens 1997; 50: 702-11.

16. LERNMARK A. Autoimmune diseases: are markers ready for prediction. J Clin Invest 2001; 108: 10916.

17. Hawa MI, Beyan H, Buckiey LR, Lesle RD. Impact of the genetic and non genetic factors in type 1 diabetes. Am J Med Genet 2002; 115: 8-17.

Agradecimientos:

Los autores agradecen la colaboración de toda la familia involucrada en esta investigación. Agradecemos también la colaboración de los Drs. Antonio Arnaiz-Villena y Jorge Martínez-Laso del Hospital Universitario 12 de Octubre, en Madrid, España. 